\title{
Electronic Imaging
}

\section{SPIEDigitalLibrary.org/jei}

\section{Quality Control by Artificial Vision}

Jean-Charles Pinoli

Karen Panetta

Seiji Hata

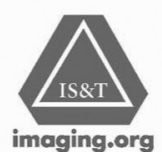




\section{Quality Control by Artificial Vision}

\author{
Jean-Charles Pinoli \\ LPMG Laboratory \\ Ecole Nationale Supérieure des Mines \\ UMR CNRS 5148 \\ 158 Cours Fauriel \\ 42023 Saint-Etienne Cedex 2, France
}

Karen Panetta

Tufts University

Department of Electrical and Computer Engineering

161 College Avenue

Medford, Massachusetts 02155

\section{Seiji Hata}

Kagawa University, Faculty of Engineering

1-1 Saiwai-cho

Takamatsu 760-8526, Japan

Over the past two decades, machine (artificial) vision systems have effectively automated many manual tasks whose goals may be achieved by the analysis and interpretation of visual data. As a result, such tasks are performed more rapidly and with much higher degrees of accuracy, precision, and repeatability.

The rapid proliferation of machine vision systems is a direct result of the tremendous advances that have been made in the research and development of:

(1) smart image acquisition subsystems,

(2) powerful mathematical and computational imaging approaches,

(3) sophisticated image processing algorithms,

(4) adaptive pattern analysis and interpretation techniques, and

(5) fast, efficient, and cost-effective data processing software and hardware.

As the advancements in each of these areas continue, the application domain of machine vision systems grows, giving rise to new opportunities and challenges.

The papers in this special section were selected from the best presentations at the annual SPIE conference on Quality Control by Artificial Vision, held June 2011 in Saint-Etienne, France.

The authors of those top-ranked presentations were invited to submit an expanded version of their conference presentation to this special section. We are delighted that the majority of those invited agreed.

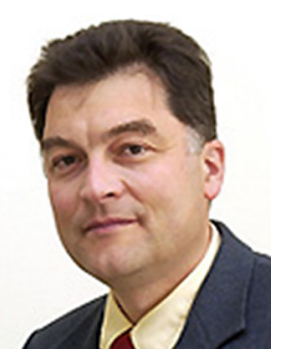

Jean-Charles Pinoli received his MSc degree in mathematics in 1982, his DSc degree in computer sciences in 1987, and his Habilitation degree in applied mathematics in 1992. From 1985 to 1989 , he was member of the opto-electronics department of the Thales-Angénieux Company, Saint-Héand, France, where he pioneered research in the field of digital imaging and artificial vision. In 1990, he joined the Corporate Research Center of the Pechiney Company, Voreppe, France and was in charge of the imaging activities within the computational technologies division. Since 2001, he has been a full professor at the French graduate school 'Ecole Nationale Supérieure des Mines de Saint-Étienne.' He leads the Image and Pattern Analysis and Modeling Group. His research interests and teaching include image processing, analysis, and modeling, pattern analysis, and computer vision, mainly focusing on the mathematical and computational aspects.

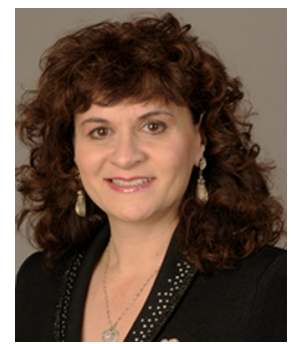

Karen Panetta received her BSC from Boston University, her MS from Northeastern University, and her $\mathrm{PhD}$ from Northeastern University (1994). She is a full professor of electrical and computer engineering at Tufts University, Medford, MA. She is an IEEE Fellow, NASA Langley Research Scientist Fellow, and recipient of the NSF CAREER Award, Norm Augustine Award from the American Association of Engineering Societies, and the Anita Borg Institute, Women of Vision Award. In 2011, United States President Barack Obama awarded her with the nation's highest award for engineering and science education and mentoring. Before joining the faculty at Tufts University, she was employed as a principal computer architect at Digital Equipment Corporation. Her current research areas include linear image processing techniques for applications in security and biomedical imaging.

Seiji Hata: biography and photograph not available. 